\title{
Die politische Repräsentation von Frauen und der Umbau des Sozialstaats
}

\author{
Lea Elsässer • Armin Schäfer
}

\author{
Article by an MPIfG researcher \\ Lea Elsässer, Armin Schäfer: Die politische Repräsentation von Frauen und der Umbau des Sozialstaats. In: Politische \\ Vierteljahresschrift 59(4), 659-680 (2018). Springer \\ The original publication is available at the publisher's web site: https://doi.org/10.1007/s11615-018-0108-6
}

Online publiziert: 25. September 2018

(C) Deutsche Vereinigung für Politikwissenschaft 2018

Zusammenfassung In diesem Aufsatz fragen wir, ob der Übergang zum Sozialinvestitionsstaat durch die wachsende Anzahl von weiblichen Abgeordneten in 21 OECD-Staaten erklärt werden kann. Damit schließen wir an Studien an, die einen positiven Zusammenhang zwischen der Repräsentation von Frauen und den Sozialausgaben insgesamt festgestellt haben. Allerdings werden bislang meist stark aggregierte Ausgabenindikatoren oder ausschließlich soziale Dienstleistungen untersucht. Wir verwenden disaggregierte Daten und betrachten sowohl sozialinvestive als auch passive Leistungen. Das Zusammenspiel von einer besseren deskriptiven politischen Repräsentation mit einer höheren Frauenerwerbsquote hilft, den Sozialstaatsumbau der letzten Jahre zu verstehen.

Schlüsselwörter Deskriptive Repräsentation · Sozialausgaben ·

Sozialinvestitionsstaat

\section{The Political Representation of Women and Welfare State Restructuring}

Abstract This article explores the relationship between the political representation of women and welfare state change in 21 OECD countries. Several authors have argued that a higher share of female parliamentarians leads to an increase in social

Zusatzmaterial online Zusätzliche Informationen sind in der Online-Version dieses Artikels (https:// doi.org/10.1007/s11615-018-0108-6) enthalten.

\author{
L. Elsässer \\ Universität Duisburg-Essen, Duisburg, Deutschland \\ E-Mail: lea.elsaesser@uni-due.de
}

\section{A. Schäfer $(\bowtie)$}

Westfälische Wilhelms-Universität, Münster, Deutschland

E-Mail: aschaefer@uni-muenster.de 
expenditure, in particular when labor market participation of women is high. However, existing studies focus on highly aggregated social expenditure measures or on social services alone. We can show that most countries have implemented both cutbacks in traditional decommodification measures and an expansion of activating social policies. The interplay of women's descriptive representation and rising female employment helps to understand this welfare state change in recent decades.

Keywords Descriptive representation $\cdot$ Women $\cdot$ Social investment state $\cdot$ Social expenditure

\section{Einleitung}

Macht es einen Unterschied für die dort getroffenen Entscheidungen, wer in den Parlamenten sitzt? Spätestens seit der Veröffentlichung von Anne Phillips Monografie The Politics of Presence (1995) wird der Zusammenhang zwischen deskriptiver und substanzieller Repräsentation von Frauen immer wieder empirisch untersucht (z.B. Brunsbach 2011; Meyer 2003). Seit einigen Jahren wird dabei verstärkt der Frage nachgegangen, ob mehr Frauen in den Parlamenten auch dazu führen, dass der Sozialstaat ausgebaut wird. Orientierte sich dieser lange Zeit an den Bedürfnissen von Männern, die Vollzeit im industriellen Sektor beschäftigt waren, haben der Aufstieg des Dienstleistungssektors und der Wandel von Familienstrukturen soziale Risiken hervorgebracht, die durch die klassischen Sicherungssysteme schlecht abgedeckt werden und von denen überproportional Frauen betroffen sind (Taylor-Gooby 2004).

Die Herausforderung, den Sozialstaat an diese Veränderungen anzupassen, fällt in eine Phase, in der die Austeritätszwänge zugenommen haben (Busemeyer et al. 2018). Zwischen 1960 und dem Ende der Achtzigerjahre stiegen die Sozialausgaben, wenngleich in unterschiedlicher Geschwindigkeit, sodass am Ende dieses Zeitraums Esping-Andersen (1990) von drei „Welten des Wohlfahrtskapitalismus“ sprechen konnte. Doch seither ist der Ausbau des Sozialstaats nach und nach zum Erliegen gekommen, und nach einer Phase der Stagnation der Ausgaben setzte ab Mitte der Neunzigerjahre ein Um- und partieller Rückbau sozialer Sicherung ein, der als Übergang zum ,Sozialinvestitionsstaat“ bezeichnet wird (Van Kersbergen und Hemerijck 2012). Charakteristisch für diesen Übergang ist der Rückbau von dekommodifizierenden Maßnahmen bei gleichzeitigem Fokus auf eine umfassende Inklusion breiter Bevölkerungsteile in den Arbeitsmarkt (Gingrich und Ansell 2015).

In diesem Kontext sind in allen entwickelten Sozialstaaten in den letzten Jahren sozialpolitische Reformen verabschiedet worden, die die Partizipation von Frauen und insbesondere von Müttern - am Arbeitsmarkt fördern und die Vereinbarkeit von Familie und Beruf ermöglichen sollen. Die jeweiligen Programme variieren stark in ihrer Ausgestaltung und dem Zeitpunkt ihrer Implementierung, folgen aber in ihrem Entstehungsprozess einem gemeinsamen Muster: Es sind überall mehrheitlich weibliche Abgeordnete, welche die Reformen initiiert und umgesetzt haben - aus ganz unterschiedlichen Parteien und nicht selten gegen die eigenen Mitglieder (Morgan 
2013). ${ }^{1}$ Dies deutet darauf hin, dass die gestiegene Repräsentation von Frauen in den Parlamenten zu sozialpolitischen Reformen führt, die die Vereinbarkeit von Familie und Beruf stärker adressieren. Gleichzeitig kann diese Entwicklung nicht isoliert betrachtet werden, sondern muss im Kontext des Umbaus des Sozialstaats verstanden werden, der die Verteilungswirkung der sozialen Sicherung verändert. Während in den letzten Jahrzehnten Transferleistungen häufig gekürzt wurden, fand ein Ausbau sowohl familienpolitischer Leistungen als auch einer aktivierenden Sozialpolitik statt.

In diesem Aufsatz argumentieren wir, dass die deskriptive politische Repräsentation von Frauen im Zusammenspiel mit der höheren Erwerbsbeteiligung von Frauen einen wesentlichen Beitrag zur spezifischen Ausgestaltung des Umbaus in den von uns untersuchten OECD-Ländern leistet. Seit den 1980er-Jahren nimmt die Anzahl weiblicher Abgeordneter zu. Waren 1980 durchschnittlich nur sechs Prozent der Abgeordneten Frauen, stieg der Anteil bis 2014 auf 31 Prozent. ${ }^{2}$ Aus Veränderungen der deskriptiven Repräsentation folgt zwar nicht automatisch ein Politikwandel, aber - so ist überzeugend normativ argumentiert (Phillips 1995) und empirisch nachgewiesen worden (im Überblick Wängnerud 2009) - die Wahrscheinlichkeit steigt, dass bislang nicht adressierte Themen angesprochen und andere Entscheidungen getroffen werden.

Bisherige Studien, welche die Implikationen deskriptiver politischer Repräsentation von Frauen untersuchen, fokussieren meist auf die Gesamtausgaben für soziale Sicherung oder nehmen nur familienpolitische Maßnahmen in den Blick. Unsere empirische Untersuchung geht darüber hinaus und zeigt, dass das Zusammenspiel aus einer hohen Erwerbsbeteiligung von Frauen bei gleichzeitiger politischer Repräsentation in den Parlamenten dazu führt, dass sozialinvestive Leistungen stärker ausgebaut werden - gleichzeitig werden absichernde Transferleistungen häufig dort besonders stark gekürzt, wo Dienstleistungen ausgebaut werden. Da jedoch (teilweise) unterschiedliche Gruppen von diesen beiden Leistungsarten profitieren, entsteht ein spezifisches Muster sozialer Ungleichheit: Während die neuen familienpolitischen Leistungen insbesondere der Mittelschicht und gut qualifizierten Frauen zugutekommen - wodurch innerhalb dieser Gruppe mehr Geschlechtergerechtigkeit möglich wird -, profitieren weniger gut qualifizierte Gruppen nicht nur weniger stark von dieser Ausweitung, sondern sind zugleich mit der Kürzung von anderen, für sie wichtigen sozialen Leistungen konfrontiert.

\footnotetext{
${ }^{1}$ Inwieweit dieser Sozialstaatsumbau tatsächlich zu mehr Geschlechtergerechtigkeit beiträgt, ist umstritten. So wird beispielsweise argumentiert, dass ursprünglich feministische Forderungen nach mehr Geschlechtergerechtigkeit in den neueren politischen Diskursen um Aktivierung und Vereinbarkeit von Familie und Beruf häufig instrumentalisiert werden, um möglichst viele Frauen in den Arbeitsmarkt zu integrieren, ohne gleichzeitig die geschlechtliche Arbeitsteilung in Bezug auf Sorge- und Hausarbeit zu verändern oder Geschlechtergleichheit als explizites Ziel zu formulieren (Jenson 2015). Ökonomische Unabhängigkeit von einem männlichen „Versorger“ bzw. die Möglichkeit, einen eigenen Haushalt gründen und führen zu können (Orloff 2006), ist allerdings ein feministisches Kernanliegen und kann als Grundvoraussetzung für Geschlechtergleichheit begriffen werden. Ein Ausbau politischer Maßnahmen, die dieses Anliegen ermöglichen, werden deshalb hier als ,frauenfreundlich“ bewertet, auch wenn auf die konkreten Verteilungsfolgen später noch näher eingegangen wird.

2 Diese Angaben sind dem CPDS (Comparative Political Data Set) entnommen (siehe Armingeon et al. 2016).
} 
Der vorliegende Artikel ist wie folgt aufgebaut. Im nächsten Abschnitt diskutieren wir, warum in der Debatte über den Wandel des Sozialstaats die besondere Bedeutung der deskriptiven politischen Repräsentation von Frauen nicht angemessen erfasst wird. Ausgeblendet wird in bisherigen Studien zumeist, dass sich die Dynamik für unterschiedliche Ausgabenarten unterscheidet, wodurch Kürzungen und Ausgabensteigerungen gleichzeitig stattfinden können. Im dritten Abschnitt stellen wir daher unsere zwei abhängigen Variablen vor, mit denen wir ,passive“ und ,sozialinvestive“ oder ,aktivierende“ Maßnahmen unterscheiden. ${ }^{3}$ Anschließend wird im vierten Abschnitt zuerst deskriptiv der Wandel des Sozialstaats anhand dieser beiden Dimensionen nachgezeichnet, bevor mithilfe von multivariaten Regressionsanalysen geprüft wird, ob die Erwerbsbeteiligung von Frauen im Zusammenspiel mit ihrer parlamentarischen Repräsentation die spezifischen Veränderungstrends erfassen helfen. Der letzte Abschnitt resümiert die Argumentation und diskutiert die Verteilungswirkungen der sozialstaatlichen Umbauprozesse.

\section{Der Übergang zum Sozialinvestitionsstaat}

Der Umbau (west-)europäischer Wohlfahrtsstaaten der letzten Jahrzehnte folgte, wenn auch mit erheblicher zwischenstaatlicher Variation, einem gemeinsamen Trend: der Entwicklung zum sogenannten „Sozialinvestitionsstaat“ (Bonoli 2013; Esping-Andersen 2002; Palier 2006; Van Kersbergen und Hemerijck 2012). Diese Entwicklung ist sowohl durch eine Veränderung der sozialpolitischen Ziele als auch durch konkrete Maßnahmen und Programme charakterisiert. Der Fokus wohlfahrtsstaatlicher Politik im Sozialinvestitionsstaat liegt nicht länger auf der sozialen Absicherung des (männlichen) Arbeiters vor Arbeitslosigkeit, Krankheit und Alter, sondern auf der Aktivierung und Inklusion möglichst großer Bevölkerungsteile in den Arbeitsmarkt. Mit dem Fokus auf Beschäftigung und der Förderung von Beschäftigungsfähigkeit sollen die Arbeitsmarktchancen benachteiligter Gruppen erhöht und gleichzeitig die Einnahmebasis des Sozialstaats verbessert werden (Bonoli 2013, S. 2). Diese Restrukturierung zum Sozialinvestitionsstaat geht gleichzeitig mit Kürzungen und einem Ausbau sozialpolitischer Programme einher. Während Kürzungen im Bereich der Arbeitslosenhilfe, wie beispielsweise die Verschärfung der Voraussetzungen für den Bezug von Sozialleistungen, Anreize zur Beschäftigung erhöhen sollen, zielt der Ausbau sozialstaatlicher Leistungen darauf, die Partizipation am Arbeitsmarkt zu ermöglichen (Palier 2006, S. 108-111). Unter sozialinvestiven Leistungen oder auch aktivierender Sozialpolitik werden hauptsächlich Maßnahmen

\footnotetext{
3 Wir verwenden diese Begriffe, weil sie in der einschlägigen Literatur verwendet werden. Die häufig mitschwingende Wertung, dass aktivierende Leistungen passiven Transfers vorzuziehen sind, teilen wir jedoch nicht. Die Begriffe markieren eine analytische, keine normative Unterscheidung.
} 
zur Vereinbarkeit von Familie und Beruf, frühkindliche Bildung und Betreuung sowie aktive Arbeitsmarktpolitik gefasst. ${ }^{4}$

Inwiefern dieser Trend in allen Wohlfahrtsstaatsregimen gleichermaßen zu beobachten ist und welche Erklärungsfaktoren dieser Entwicklung zugrunde liegen, wird unterschiedlich beantwortet. Palier (2006) beispielsweise sieht in den veränderten makroökonomischen Rahmenbedingungen einen Hauptgrund für die Restrukturierung zum Sozialinvestitionsstaat. Nach der Abkehr von keynesianischer Wirtschaftspolitik in den 1970ern musste auch die Sozialpolitik dem neuen, angebotsorientierten wirtschaftspolitischen Paradigma angepasst werden. Ausgabensenkungen und Beschäftigungsförderung wurden Hauptziele der neuen sozialpolitischen Ausrichtung, was zur Entstehung des ,social investment state“ führte (Palier 2006, S. 105-106). Der Fokus auf Beschäftigungsförderung - insbesondere von Frauen - hat demnach auch das Ziel, die Sozialsysteme durch die Verbreiterung der Steuerbasis finanziell zu stabilisieren (Palier 2006, S. 108-111). Der Wandel zum Sozialinvestitionsstaat wird hier zu einem großen Teil an fiskalpolitischen Notwendigkeiten oder Zwängen festgemacht (Bonoli 2013, S. 4; Pierson 2001).

Eine Reihe von anderen Beiträgen nimmt nicht primär die wirtschaftspolitischen Möglichkeiten des Staats, sondern die sich verändernde Nachfrage nach sozialpolitischen Maßnahmen in den Blick. Autorinnen und Autoren aus diesem Bereich argumentieren, dass durch die sozioökonomischen und demografischen Veränderungen seit den 1970er-Jahren ,neue soziale Risiken“ entstanden sind, die einen Bedarf an neuen Formen der Sozialpolitik nach sich ziehen (Bonoli 2007; Esping-Andersen 1999; Taylor-Gooby 2004). So erhöhen veränderte Arbeitsmarktbedingungen im Zuge von De-Industrialisierung, der Ausweitung des Dienstleistungssektors und der Erosion des Normalarbeitsverhältnisses das Risiko unsicherer und niedrig entlohnter Beschäftigung sowie das Risiko von (Langzeit-)Arbeitslosigkeit und dadurch einer unzureichenden sozialer Absicherung für Menschen mit unsteten Erwerbsbiografien (Bonoli 2007, S. 498-499). Der Eintritt von Frauen in den Arbeitsmarkt und weniger stabile familiäre Bindungen erschweren die Vereinbarkeit von Familie und Beruf und erhöhen den Bedarf an Kinderbetreuungseinrichtungen und außerfamiliären Pflegeleistungen (Bonoli 2007, S. 499-500; Taylor-Gooby 2004, S. 2-5). Besonders von diesen Risiken betroffen sind Frauen, junge Menschen und prekär Beschäftigte im unteren Dienstleistungssektor, die von dem am männlichen Alleinverdiener ausgerichteten Sozialstaat nicht ausreichend abgedeckt werden. Bonoli (2007) argumentiert, dass der Zeitpunkt dieser sozioökonomischen Veränderungen in den jeweiligen Wohlfahrtsstaaten ausschlaggebend dafür war, ob und in welchem Umfang Sozialpolitik responsiv gegenüber dieser neuen Nachfrage sein konnte. Vollzogen sich die Veränderungen schon früh und in Zeiten, in denen der Wohlfahrtsstaat noch in seiner expansiven Phase war, so konnten sich auch Maßnahmen zur Abfederung neuer sozialer Risiken besser durchsetzen, da der Kostendruck auf die immer teurer

\footnotetext{
${ }^{4}$ Einige Autorinnen und Autoren zählen auch Investitionen in Bildungspolitik zu sozialinvestiven Maßnahmen, siehe z. B. Nikolai (2012), da die (Aus-)Bildung starken Einfluss auf spätere Arbeitsmarktchancen hat und somit unter „Humankapitalinvestitionen“ gefasst werden kann. Da es in diesem Beitrag aber vornehmlich um die Umstrukturierung und Verschiebung von Ausgaben und Maßnahmen innerhalb des enger gefassten - Bereichs der Sozialpolitik geht, sind bildungspolitische Maßnahmen nicht Gegenstand unserer Betrachtungen.
} 
werdenden wohlfahrtsstaatlichen Systeme noch nicht so hoch war. Daneben wird auch argumentiert, dass die verschiedenen wohlfahrtsstaatlichen Regime aufgrund ihrer institutionellen Voraussetzungen unterschiedlich stark auf die neue Nachfrage reagieren (Taylor-Gooby 2004) und der Ausbau ,aktiver Sozialpolitik“ zudem davon abhängt, inwieweit die politischen Entscheidungsträgerinnen und -träger in Zeiten von Rückbau und Austerität durch die Einführung aktiver Sozialpolitik ,credit claiming" für sich beanspruchen können (Bonoli 2013).

Die bisher genannten Erklärungsansätze eint, dass sie eine zwingende Notwendigkeit einer Restrukturierung des Sozialstaats und damit eine beinahe automatische Anpassung wohlfahrtsstaatlicher Institutionen und Leistungen an veränderte wirtschaftliche und gesellschaftliche Rahmenbedingungen annehmen. Auch wenn politökonomische und gesellschaftliche Veränderungen bedeutende strukturelle Faktoren für die Erklärung sozialstaatlichen Wandels sind, bleiben die konkreten politischen Mechanismen sowie Akteurinnen und Akteure, die diesen Wandel forcieren, in diesen Erklärungsansätzen häufig unberücksichtigt. Stärker akteurszentrierte Ansätze dagegen legen nahe, dass der Umbau in starkem Zusammenhang mit einer Akteursgruppe steht, die beim Aufbau des Wohlfahrtsstaats alter Prägung nur eine untergeordnete Rolle gespielt hat: Frauen.

In den letzten dreißig Jahren hat sich die Erwerbsbeteiligung von Frauen in den OECD-Staaten deutlich erhöht. War 1980 nur etwa jede zweite Frau erwerbstätig, sind es heute im Durchschnitt mehr als zwei Drittel, in manchen Ländern sogar drei Viertel aller Frauen. Durch die stärkere Arbeitsmarktteilnahme nehmen Vereinbarkeitsprobleme von Familie und Beruf zu. Da Hausarbeit, Kinderbetreuung und Pflege noch immer ungleich zwischen Frauen und Männern verteilt sind, sollten insbesondere Frauen an einer öffentlichen Infrastruktur interessiert sein, die die Vereinbarkeit erleichtert (Huber und Stephens 2000, S. 327). Daher sollten sich vor allem erwerbstätige Frauen für den Ausbau sozialer Leistungen in diesen Bereichen einsetzen und Parteien wählen, die diese Ziele unterstützen. Der ,modern gender gap“ (Inglehart und Norris 2003, S. 78) stützt diese Annahme: Während in den 1950er- und 1960er-Jahren Frauen stärker als Männer konservative Einstellungen aufwiesen und eher konservative Parteien wählten, hat sich dieses Muster in den postindustriellen Gesellschaften und jüngeren Altersgruppen ins Gegenteil verkehrt (Giger 2009; Inglehart und Norris 2003). Insbesondere erwerbstätige Frauen befürworten staatliche Eingriffe, die es ihnen ermöglichen, Beruf und Betreuung miteinander zu verbinden (Finseraas, Jakobsson und Kotsadam 2012, S. 227; Iversen und Rosenbluth 2006, S. 15-16).

Die gestiegene politische Nachfrage nach Maßnahmen zur Vereinbarkeit von Familie und Beruf führt jedoch nur dort zu politischen Veränderungen, wo Frauen in den Parlamenten in ausreichendem Maß repräsentiert sind. So zeigen Bratton und Ray (2002), dass eine höhere Anzahl von weiblichen Mitgliedern in norwegischen Gemeinderäten die Anzahl von Kitaplätzen erhöht. Kittilsons (2008) Analyse von Daten aus 19 Ländern zwischen 1970 und 2000 bestätigt ebenfalls, dass eine höhere Anzahl weiblicher Abgeordneter zu großzügigeren Mutterschutz- und Elternzeitregelungen führt. Unabhängig von Parteizugehörigkeit, Alter, Bildungsgrad und der Dauer der Parlamentszugehörigkeit, so verdeutlicht Wängneruds (2000) Auswertung mehrerer Befragungen von schwedischen Parlamentarierinnen und Parlamentariern, 
schätzen weibliche Abgeordnete häufiger als ihre männlichen Kollegen sozialpolitische Anliegen als besonders wichtig ein. Diese Arbeiten legen nahe, dass es das Zusammenspiel aus einer gestiegenen Nachfrage mit der besser deskriptiven Repräsentation von Frauen ist, die zu höheren Sozialausgaben, die die Vereinbarkeit von Erwerbstätigkeit und Betreuungsanforderungen erhöhen, führen.

Die Ergebnisse einer Reihe von Studien sprechen für diesen Zusammenhang. Huber und Stephens (2000) zeigen, dass die Ausgaben für soziale Dienstleistungen und die Arbeitsplätze im öffentlichen Sektor steigen, wenn die Erwerbsbeteiligung von Frauen zunimmt, und führen dies darauf zurück, dass lohnarbeitende Frauen verstärkt soziale Dienstleistungen nachfragen. Dieser Effekt zeigt sich besonders stark unter sozialdemokratischen Regierungen, weshalb sie argumentieren, dass das Zusammenspiel aus linksgerichteten Regierungen und der politischen Mobilisierung von Frauen in linken Parteien den Ausbau von sozialen Dienstleistungen begünstigt (Huber und Stephens 2000, S. 335). In ihrer Analyse der Sozialausgaben insgesamt finden Bolzendahl und Brooks (2007) einen besonders starken positiven Einfluss der Frauenerwerbsquote auf die Sozialausgaben, wenn gleichzeitig die Anzahl von Frauen im Parlament hoch ist. Durch den Fokus auf die aggregierten Sozialausgaben können sie allerdings nicht unterscheiden, inwiefern die politische Repräsentation von Frauen eine generelle Erweiterung oder einen Umbau des Sozialstaats zur Folge hat. Eine weitere Studie von Bolzendahl (2011) legt zwar nahe, dass vor allem der Ausbau von Dienstleistungen von Parlamentarierinnen vorangetrieben wird - hier wird allerdings nicht das Zusammenspiel von Erwerbsbeteiligung und politischer Repräsentation betrachtet. ${ }^{5}$ Zudem endet der Untersuchungszeitraum bereits 1999, obwohl der Ausbau sozialinvestiver Maßnahmen in vielen Staaten erst seit Mitte der 1990er-Jahre begonnen hat (Bonoli 2013). In Bezug auf aktive familienpolitische Maßnahmen argumentieren Ferragina und Seeleib-Kaiser (2015), dass der Einfluss weiblicher Abgeordneter auf den Ausbau dieser Programme in den 1980er- und 1990er-Jahren hoch war, seit den 2000ern aber an Bedeutung verloren hat. Stattdessen sei der Ausbau seit den 2000er-Jahren stärker von einer allgemeinen gesellschaftlichen Zustimmung zu „modernen“ Familien- und Lebensentwürfen getragen, die aber wiederum auch durch die politische Mobilisierung von Frauen herbeigeführt worden sei (Ferragina und Seeleib-Kaiser 2015, S. 25). Mithilfe detaillierter Fallstudien zeigt Morgan (2013) für Deutschland, Großbritannien und die Niederlande, dass ein verstärkter Wettbewerb um die Stimmen weiblicher Wählerinnen und eine verstärkte Repräsentation von Frauen in politischen Parteien und Regierungsposten dazu geführt hat, dass in allen drei Staaten Maßnahmen zur Vereinbarkeit von Familie und Beruf ausgebaut wurden.

Der Blick auf das Zusammenspiel von Frauenerwerbsquote und deskriptiver Repräsentation leistet einen wichtigen Beitrag zum Verständnis von sozialpolitischen Ausgabentrends und Reformen. Allerdings werden meist entweder stark aggregierte Ausgabenindikatoren oder ausschließlich familienpolitische Maßnahmen untersucht, ohne sie in den Kontext eines generellen Wohlfahrtsstaatsumbaus einzubetten und

\footnotetext{
5 Tepe und Vanhuysse (2010) finden keinen Einfluss weiblicher Parlamentarierinnen auf die Höhe der Sozialausgaben, was aber daran liegen kann, dass sie in ihrer Untersuchung verschiedener Ausgabenbereiche keine Unterscheidung zwischen aktiver und passiver bzw. Transfer- und Dienstleistungen vornehmen.
} 
Kürzungen in anderen Bereichen mit zu betrachten (Bolzendahl und Brooks 2007; Ferragina und Seeleib-Kaiser 2015; Huber und Stephens 2000; Morgan 2013). Dadurch treten die Erklärungen genereller Umstrukturierungsprozesse und politischer Implikationen in den Hintergrund. Zudem werden Frauen in diesen Erklärungsansätzen als homogene Gruppe betrachtet und ihnen damit - zumindest implizit einheitliche Interessen unterstellt. Das dahinterliegende Argument ist, dass sie besonders stark von „,neuen sozialen Risiken“ und von Geschlechterungleichheit am Arbeitsmarkt und in der Familie betroffen sind und deshalb sowohl sozialinvestive Maßnahmen verstärkt nachfragen als auch als politische Akteurinnen durchsetzen. Auch wenn dies zweifelsohne richtig ist, so scheint die Annahme einer homogenen Gruppe bei genauer Betrachtung problematisch, denn neben dem Geschlecht spielt auch die soziale Klasse eine zentrale Rolle bei der Frage, wer von „,neuen“ und „alten“ sozialen Risiken betroffen ist (Pintelon et al. 2013). So sind beispielsweise das Risiko, arbeitslos zu werden, aber auch die Scheidungsraten und der Anteil alleinerziehender Mütter in unteren Einkommensschichten deutlich höher - und damit auch das Armutsrisiko (Esping-Andersen 2009, S. 66-67; Pintelon et al. 2013).

Wie diese Ausführungen zeigen, ist eine gemeinsame Betrachtung verschiedener Bereiche sozialer Sicherung notwendig, um die Bedeutung deskriptiver politischer Repräsentation für wohlfahrtsstaatlichen Wandel zu verstehen. In diesem Artikel soll deshalb untersucht werden, wie sich die politische Repräsentation von Frauen auf den beschriebenen Sozialstaatsumbau - also auf die Veränderungen in sozialinvestiven und passiven Staatsausgaben - auswirkt. Damit geht unsere Analyse über bisherige Untersuchungen hinaus, die sich weitestgehend auf den Einfluss politischer Repräsentation von Frauen auf die Gesamtausgaben oder auf soziale Dienstleistungen beschränken.

\section{Daten und Methode}

Im Folgenden untersuchen wir Ausgabenentwicklungen in 21 OECD-Staaten im Zeitraum von 1980 bis 2011.6 Dabei schließen wir jene OECD-Staaten ein, die im gesamten Zeitraum demokratisch regiert wurden und für die die benötigten Daten vorliegen. Die zwei Hauptindikatoren unserer Analyse sind die öffentlichen Ausgaben für passive und für aktivierende Sozialpolitik (in Prozent des Bruttoinlandsprodukts). Die Daten stammen aus der „OECD Social Expenditure Database“ (OECD 2016), in welcher die Ausgaben für verschiedene sozialpolitische Bereiche - aufgeschlüsselt nach einzelnen Programmen und der Unterscheidung in Dienst- und Geldleistungen - separat abgerufen werden können. Mithilfe dieser disaggregierten Ausgabendaten konnten die folgenden zwei Ausgabenkategorien gebildet werden. Die öffentlichen Ausgaben für aktivierende Sozialpolitik enthalten Dienstleistungen für Familien, Ausgaben für Elterngeld bzw. Mutterschutz, Pflegeleistungen für alte und berufsunfähige Menschen und Ausgaben für aktive Arbeitsmarktpolitik (siehe

\footnotetext{
${ }^{6}$ Die in den Analysen verwendeten Länder sind Australien, Belgien, Dänemark, Deutschland. Finnland, Frankreich, Griechenland, Irland, Italien, Japan, Kanada, Niederlande, Neuseeland, Norwegen, Österreich, Portugal, Spanien, Schweden, Schweiz, UK und USA. Untersucht werden die Jahre von 1980 bis 2011.
} 
Tab. 1 Aufteilung sozialpolitischer Ausgaben

\begin{tabular}{ll}
\hline Aktivierende Sozialpolitik & Passive Sozialpolitik \\
\hline Dienstleistung für Familien & Arbeitslosen- und Sozialhilfe \\
Zahlungen für Elternzeit/Mutterschutz & Krankengeld \\
Pflegeleistungen für Senioren und Berufsunfähige & Rente bei Berufsunfähigkeit \\
Aktive Arbeitsmarktpolitik & Kindergeld und andere Transferzahlungen an \\
& Familien \\
\hline
\end{tabular}

Tab. 1). Unter die Dienstleistungen für Familien fallen hauptsächliche frühkindliche Erziehung und Betreuung. Sie gehören - zusammen mit Elternzeit und Mutterschutz - zu den Kernmaßnahmen zur Förderung der Vereinbarkeit von Familie und Beruf. ${ }^{7}$ Pflegeleistungen für Senioren und Berufsunfähige fallen ebenfalls in diese Kategorie, da die Pflege von Angehörigen für viele (vor allem ältere) Frauen ein Ausscheiden aus dem Arbeitsmarkt bedeutet, wenn sie keine staatlichen Pflegeleistungen in Anspruch nehmen können (Esping-Andersen 2009, S. 97-99). Ausgaben für aktive Arbeitsmarktpolitik sind für die meisten Staaten erst ab 1990 verfügbar und deshalb ab diesem Zeitpunkt in die Analysen integriert. Die öffentlichen passiven sozialpolitischen Ausgaben umfassen die Maßnahmen zur Absicherung gegen ,traditionelle" Risiken: Ausgaben für Arbeitslosen- und Sozialhilfe, Krankengeld und Berufsunfähigkeitsrenten sowie Transferzahlungen für Familien (Kindergeld etc.). Rentenausgaben wurden nicht in die Indikatoren mit einbezogen, da wir bei der Gegenüberstellung von aktivierender und passiver Sozialpolitik hauptsächlich Verschiebungen innerhalb der Ausgaben für die Bevölkerung im arbeitsfähigen Alter betrachten wollen.

Die zentrale These unseres Beitrags lautet, dass der Übergang zum Sozialinvestitionsstaat einerseits durch gesellschaftliche Veränderungen angestoßen wird, die die Nachfrage insbesondere nach sozialen Dienstleistungen erhöhen, und andererseits nur dann stattfindet, wenn es politische Akteurinnen und Akteure gibt, die ihn unterstützen. Da dieser Umbau des Sozialstaats in Zeiten fiskalischer Austerität stattfindet, werden nicht einfach bestehende Leistungen ergänzt, sodass die Gesamtausgaben steigen, sondern Kürzung und Ausweitung finden gleichzeitig statt.

Um das Zusammenspiel von Nachfrage und politischer Repräsentation einzufangen, schließen wir in unserer Analyse einerseits die Frauenerwerbsquote und andererseits die Anzahl weiblicher Abgeordneter ein, wie dies auch in anderen Studien geschieht (Bolzendahl und Brooks 2007). Zusätzlich werden Variablen beachtet, die den Bedarf an Sozialleistungen beeinflussen, wie etwa der Anteil Älterer oder von Kindern an der Gesamtbevölkerung sowie die Arbeitslosenquote. Wahlbeteiligung, der kumulierte Anteil von linken Regierungen und die Gewerkschaftsstärke erfassen klassische Indikatoren der vergleichenden Sozialpolitikforschung. Schließlich werden mit dem Außenhandel und dem BIP-Wachstum die ökonomischen Rahmen-

\footnotetext{
7 Wie die einzelnen Politikmaßnahmen ausgestaltet sind - bspw. in Bezug auf die Dauer, das Anspruchsrecht und die Höhe der Leistungen - geht aus den Ausgabendaten selbstverständlich nicht hervor, weshalb auch allein von der Höhe der Ausgaben nicht auf konkrete politische Ergebnisse geschlossen werden kann. Trotzdem gehen wir davon aus, dass höhere Ausgaben in diesen Bereichen auch eine höhere politische Priorität für die Ziele aktivierender Sozialpolitik widerspiegeln.
} 
bedingungen beachtet, während die Variable „Machtverteilung“ misst, inwieweit ein politisches System durch Vetopunkte geprägt ist. ${ }^{8}$ Da die skandinavischen Länder durch den hohen Anteil an sozialinvestiven Ausgaben hervorstechen, benutzen wir in einigen Modellen eine Dummy-Variable für diese Ländergruppe. Schließlich überprüfen wir, ob ein zeitlicher Trend vorliegt, indem wir das Jahrzehnt als Kontrollvariable einfügen.

Das von uns verwendete Regressionsmodell kombiniert ,panel corrected standard errors“ (PCSE) mit einer Prais-Winsten-Transformation (AR1), um für Autokorrelation in den Störtermen zu kontrollieren (Beck 2001; Beck und Katz 1995). ${ }^{9}$ Wir verwenden Fixed effects-Modelle nur zur Kontrolle, da wir annehmen, dass die im Fokus stehenden erklärenden Variablen - Frauenerwerbsquote und Anzahl weiblicher Abgeordneter - Leveleffekte aufweisen und somit nicht nur die Variation innerhalb eines Landes, sondern auch die Unterschiede zwischen den von uns untersuchten Ländern von Interesse sind. Dies bedeutet, dass nicht nur die Veränderung der erklärenden Variable, sondern auch ihre absolute Höhe ausschlaggebend ist. In Bezug auf die Repräsentation von Frauen gehen wir davon aus, dass weibliche Abgeordnete erst dann erfolgreich neue Themen auf die Agenda setzen und für diese Mehrheiten organisieren können, wenn ihr Anteil im Parlament eine ,kritische Masse“ erreicht (Grey 2006; Wängnerud 2009, S. 60). In solch einem Fall ist die Inklusion von Länder-Dummies (fixed effects) nicht sinnvoll, da dadurch die Variation zwischen den untersuchten Ländern herausgerechnet und lediglich der Effekt von Veränderungen innerhalb der Untersuchungseinheiten geschätzt wird (Huber und Stephens 2014; Plümper, Troeger und Manow 2005, S. 330-334).

Umstritten ist, ob es notwendig ist, die ,lagged dependent variable“ in das Modell einzuschließen, da diese theoretisch uninteressant ist - schließlich ist es fast trivial, dass sich soziale Prozesse langsam vollziehen und sich die Ausgabenhöhe eines Sozialprogramms in einem Jahr am besten durch die Ausgabenhöhe im vorangegangenen erklären lässt (Achen 2000). Methodisch empfehlen Beck und Katz (1995) dennoch diese Vorgehensweise. Wir folgen dieser Empfehlung, weil dies die konservativere Schätzvariante ist. Unsere Modelle überprüfen, welche Faktoren eine positive und negative Abweichung des allgemeinen Trends erklären, weil wir beantworten möchten, ob die (überproportionale) Zunahme an weiblichen Abgeordneten den Übergang zum Sozialinvestitionsstaat beschleunigt.

\section{Empirische Analyse: Sozialpolitik von Frauen für Frauen?}

In diesem Abschnitt untersuchen wir, wie sich die beiden Arten von Sozialausgaben entwickelt haben und ob die beobachteten Veränderungstrends durch das Zusammenspiel von einer höheren Frauenerwerbsquote mit mehr weiblichen Abgeordneten erklärt werden können. Dabei schauen wir uns zuerst deskriptiv die Ausgabentrends an, bevor anschließend multivariate Regressionsanalysen durchgeführt werden.

\footnotetext{
8 Ein Überblick über die von uns verwendeten Variablen und ihre Quellen findet sich in Tabelle A-1 im Online-Anhang.

9 Auseinandersetzungen mit dieser Methode finden sich bei Kittel (1999) sowie Wilson und Butler (2017).
} 
Gesamte Sozialausgaben für Menschen im arbeitsfähiges Alter

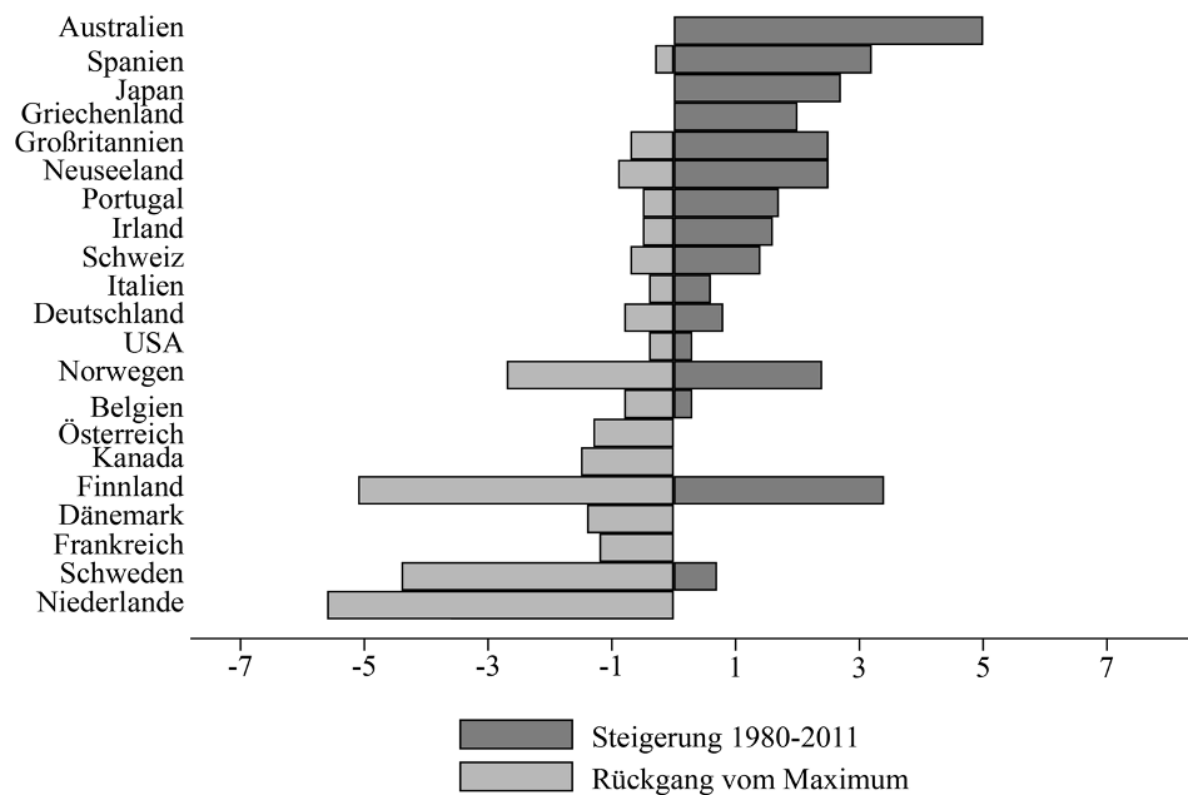

Abb. 1 Ausbau und Rückbau der Sozialausgaben zwischen 1980 und 2011. Quelle: OECD (2016). Anmerkung: Die Ausgaben werden in Prozent des Bruttoinlandsprodukts gemessen, sodass , -1 “ einen Ausgabenrückgang um einen Prozentpunkt des BIP kennzeichnet

\subsection{Entwicklung der passiven und aktivierenden Sozialausgaben}

$\mathrm{Ab}$ Mitte der Neunzigerjahre wurde diskutiert, ob ein Abbau des Sozialstaats stattgefunden habe und wie dies am besten empirisch zu ermitteln wäre. Durch die Verwendung kleinteilig aggregierter Ausgabendaten lässt sich differenziert beantworten, in welchen Bereichen Ausgaben gekürzt und wo sie sogar ausgeweitet wurden. Wir konzentrieren uns im Folgenden auf Sozialausgaben für die Bevölkerung im arbeitsfähigen Alter, da der von uns diskutierte Umbau des Sozialstaats vor allem in diesem Bereich stattfindet. Zudem sind es diese sozialen Leistungen, die zwar am meisten zur Minderung ökonomischer Ungleichheit beitragen (Castles 2009, S. 58), gleichzeitig jedoch unter besonders starkem Kürzungsdruck stehen (Elsässer et al. 2015). Wie Abb. $1^{10}$ zeigt, setzten in fast allen von uns betrachteten 21 OECD-Ländern in den letzten drei Dekaden Kürzungen bei den Sozialausgaben für die Bevölkerung im erwerbsfähigen Alter ein. Diese Ausgaben umfassen alle passiven und aktiven Leistungen zusammen, die im vorherigen Abschnitt beschrieben wurden.

\footnotetext{
${ }^{10}$ Die Grafik ist so aufgebaut, dass nach rechts weisende dunkelgraue Balken anzeigen, ob im Untersuchungszeitraum eine absolute Erhöhung der Ausgaben stattgefunden hat, also ob im Jahr $2011 \mathrm{mehr}$ ausgegeben wurde als im Jahr 1980. Die nach links weisenden hellgrauen Balken dagegen geben an, ob es zu einem Zeitpunkt vor 2011 schon einmal höhere Ausgaben gegeben hat, da sie die Ausgaben in 2011 mit dem höchsten Wert im Untersuchungszeitraum vergleichen. Sie geben also an, ob im Verlauf des Untersuchungszeitraumes Kürzungen stattgefunden haben.
} 


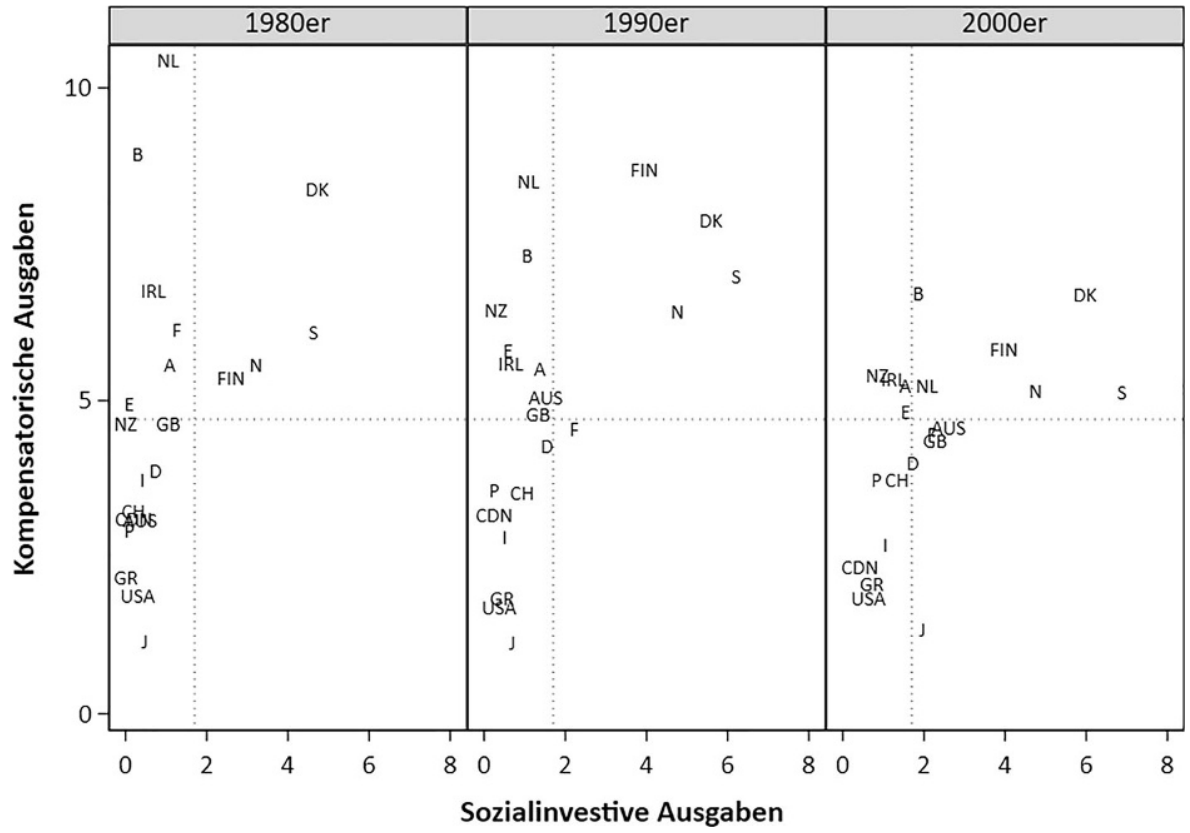

Abb. 2 Veränderungstrends bei kompensatorischen und investiven Sozialausgaben. Quelle: OECD (2016). Anmerkungen: Die Ausgaben werden jeweils in Prozent des Bruttoinlandsprodukts gemessen. Die gepunkteten Linien markieren den Durchschnitt über alle Länder und Jahre hinweg

Aus Abb. 1 lässt sich entnehmen, dass bei den Sozialausgaben nur in drei Ländern (Australien, Japan und Griechenland) keine Kürzungen stattgefunden haben. In allen anderen Ländern gab es in der Vergangenheit höhere Ausgaben als 2011. Dabei fällt auf, dass die Ausgaben im Jahr 2011 in fünf Ländern - Österreich, Kanada, Dänemark, Frankreich und den Niederlanden - unter denen des Jahres 1980 liegen. Besonders starke Rückgänge finden sich auch in Norwegen, Finnland und Schweden, sodass die skandinavischen Länder zu denen gehören, die die Sozialausgaben für die Bevölkerung im Erwerbsalter besonders stark gekürzt haben.

In Abb. 2 werden die Ausgabentrends nach aktiven und passiven Leistungen aufgeschlüsselt und Veränderungen der beiden Ausgabenarten im Zeitverlauf abgebildet. Drei Beobachtungen stechen ins Auge: Erstens haben in fast allen Ländern während des Untersuchungszeitraumes Kürzungen in den passiven Leistungen stattgefunden. In zehn Ländern liegen die heutigen Ausgaben sogar unter dem Niveau von 1980. Dagegen zeigt sich, zweitens, dass bei den aktivierenden Maßnahmen die Ausweitung dominiert. Alle 21 Länder geben heute mehr für Aktivierung aus als das 1980 noch der Fall war, und in neun Ländern ist das letzte Jahr, für das Daten verfügbar sind, auch das Jahr mit den höchsten Ausgaben. Obwohl gleichzeitig in 12 Ländern Rückgänge gegenüber dem Maximum zu verzeichnen sind, fallen diese durchweg gering aus. Drittens geht aus der Abbildung hervor, dass es eine Annäherung in den Ausgabenprofilen der 21 betrachteten Ländern gegeben hat, weil die Ausgaben für passive Leistungen insbesondere in den Ländern mit ursprünglich besonders hohen Ausgaben rückläufig sind und insgesamt eine - wenn auch 
Tab. 2 Erklärung des Umfangs von aktivierenden Sozialleistungen

\begin{tabular}{|c|c|c|c|c|}
\hline & Modell 1 & Modell 2 & Modell 3 & Modell 4 \\
\hline LDV & $\begin{array}{l}0,710 * * * \\
(0,03)\end{array}$ & $\begin{array}{l}0,665 * * * \\
(0,03)\end{array}$ & $\begin{array}{l}0,535 * * * \\
(0,04)\end{array}$ & $\begin{array}{l}0,820 \text { *** } \\
(0,03)\end{array}$ \\
\hline Frauenerwerbsquote & $\begin{array}{l}0,006^{* * * *} \\
(0,00)\end{array}$ & $\begin{array}{l}-0,007 * * \\
(0,00)\end{array}$ & $\begin{array}{l}-0,003 \\
(0,00)\end{array}$ & $\begin{array}{l}-0,004 \\
(0,00)\end{array}$ \\
\hline Parlamentarierinnen & $\begin{array}{l}0,007 * * \\
(0,00)\end{array}$ & $\begin{array}{l}-0,059 * * * \\
(0,01)\end{array}$ & $\begin{array}{l}-0,059 * * * \\
(0,01)\end{array}$ & $\begin{array}{l}-0,040^{* * * *} \\
(0,01)\end{array}$ \\
\hline Erwerbsquote*Parlamentarierinnen & - & $\begin{array}{l}0,001 \text { *** } \\
(0,00)\end{array}$ & $\begin{array}{l}0,001 * * * \\
(0,00)\end{array}$ & $\begin{array}{l}0,001 * * * \\
(0,00)\end{array}$ \\
\hline Wahlbeteiligung & $\begin{array}{l}-0,003 * \\
(0,00)\end{array}$ & $\begin{array}{l}-0,001 \\
(0,00)\end{array}$ & $\begin{array}{l}0,007 * * * \\
(0,00)\end{array}$ & $\begin{array}{l}0,004 * * \\
(0,00)\end{array}$ \\
\hline Mitte-links-Regierungen & $\begin{array}{l}0,020 * * * \\
(0,00)\end{array}$ & $\begin{array}{l}0,018^{* * * *} \\
(0,00)\end{array}$ & $\begin{array}{l}0,015^{* * * *} \\
(0,00)\end{array}$ & $\begin{array}{l}0,006 * * \\
(0,00)\end{array}$ \\
\hline Gewerkschaftsdichte & $\begin{array}{l}0,009 * * * \\
(0,00)\end{array}$ & $\begin{array}{l}0,008^{* * * *} \\
(0,00)\end{array}$ & $\begin{array}{l}-0,006 * * * \\
(0,00)\end{array}$ & $\begin{array}{l}-0,002 \\
(0,00)\end{array}$ \\
\hline Arbeitslosenquote & $\begin{array}{l}0,011 * * * \\
(0,00)\end{array}$ & $\begin{array}{l}0,015^{* * * *} \\
(0,00)\end{array}$ & $\begin{array}{l}0,016^{* * * *} \\
(0,00)\end{array}$ & $\begin{array}{l}0,014 * * \\
(0,00)\end{array}$ \\
\hline Anteil Über-65-Jährige & $\begin{array}{l}0,025 * * * \\
(0,01)\end{array}$ & $\begin{array}{l}0,036 * * * \\
(0,01)\end{array}$ & $\begin{array}{l}0,049 * * * \\
(0,01)\end{array}$ & $\begin{array}{l}0,042 * * * \\
(0,01)\end{array}$ \\
\hline Anteil Unter-5-Jährige & $\begin{array}{l}-0,006 \\
(0,02)\end{array}$ & $\begin{array}{l}0,009 \\
(0,02)\end{array}$ & $\begin{array}{l}0,020 \\
(0,02)\end{array}$ & $\begin{array}{l}0,053 * \\
(0,02)\end{array}$ \\
\hline Außenhandel (log.) & $\begin{array}{l}-0,163 * * * \\
(0,03)\end{array}$ & $\begin{array}{l}-0,140 * * * \\
(0,03)\end{array}$ & $\begin{array}{l}0,066+ \\
(0,04)\end{array}$ & $\begin{array}{l}0,088 * \\
(0,04)\end{array}$ \\
\hline BIP (log.) & $\begin{array}{l}0,263 * * * \\
(0,05)\end{array}$ & $\begin{array}{l}0,329 * * * \\
(0,06)\end{array}$ & $\begin{array}{l}0,457 * * * \\
(0,07)\end{array}$ & $\begin{array}{l}0,173 * \\
(0,08)\end{array}$ \\
\hline Vetopunkte & $\begin{array}{l}-0,027 * * * \\
(0,01)\end{array}$ & $\begin{array}{l}-0,026 * * * \\
(0,01)\end{array}$ & $\begin{array}{l}-0,015+ \\
(0,01)\end{array}$ & $\begin{array}{l}0,001 \\
(0,01)\end{array}$ \\
\hline Skandinavien & - & - & $\begin{array}{l}1,393 \text { *** } \\
(0,15)\end{array}$ & $\begin{array}{l}0,482 * * * \\
(0,14)\end{array}$ \\
\hline \multicolumn{5}{|l|}{ Jahrzehnt (RK: 1980er-Jahre) } \\
\hline $1990 \mathrm{er}$ & - & - & $\begin{array}{l}0,129 * * \\
(0,05)\end{array}$ & $\begin{array}{l}0,176^{* *} \\
(0,06)\end{array}$ \\
\hline 2000er & - & - & $\begin{array}{l}0,052 \\
(0,06)\end{array}$ & $\begin{array}{l}0,051 \\
(0,08)\end{array}$ \\
\hline Konstante & $\begin{array}{l}-2,735 * * * \\
(0,56)\end{array}$ & $\begin{array}{l}-2,978 * * * \\
(0,61)\end{array}$ & $\begin{array}{l}-5,648^{* * * *} \\
(0,75)\end{array}$ & $\begin{array}{l}-2,986^{* * * *} \\
(0,82)\end{array}$ \\
\hline $\mathrm{R}^{2}$ & 0,94 & 0,94 & 0,94 & 0,96 \\
\hline$N$ & 594 & 594 & 594 & 522 \\
\hline
\end{tabular}

Standardfehler in Klammern. $+p<0,1, * p<0,05, * * p<0,01, * * * p<0,001$

Anmerkung: Dargestellt sind die Koeffizienten einer OLS-Regression mit Panel Corrected Standard Errors mit einer Prais-Winsten-Transformation (AR1), um für Autokorrelation in den Störtermen zu korrigieren. Alle Modelle schließen die Lagged Dependent Variable (LDV) als unabhängige Variable ein.

ungleichmäßige - Ausweitung der aktivierenden Leistungen stattgefunden hat. Der Blick auf die Veränderungstrends bestätigt, dass die Sozialausgaben für die Bevölkerung im erwerbstätigen Alter insgesamt in der Mehrzahl der Länder rückläufig sind und gleichzeitig eine Umschichtung von kompensatorischen zu sozialinvestiven Ausgaben stattfinden. Das Mehr an aktiven Ausgaben ergänzt somit nicht schon 
bestehende Ausgaben, sondern geht mit einem Weniger an Leistungen in anderen Bereichen einher. ${ }^{11}$

\subsection{Beeinflussen Erwerbsbeteiligung und die politische Repräsentation von Frauen die Sozialausgaben?}

Ein Nachteil der Darstellungen im vorherigen Abschnitt ist, dass nicht beachtet wird, welche anderen Trends die gezeigten Muster erklären könnten. So kann ein Rückgang der Arbeitslosenquote die aufsummierten Ausgaben für passive Leistungen sinken lassen, ohne dass Kürzungen für Arbeitslose stattgefunden haben. Auch ein starkes Wirtschaftswachstum kann zu einer niedrigeren Ausgabenquote führen, wenn das BIP schneller als die Ausgaben wächst. Eine höhere Anzahl von Kindern im Kitaalter erhöht dagegen den Bedarf an familienpolitischen Ausgaben. Für eine Erklärung der Veränderungen bei aktivierenden und passiven Ausgaben müssen also weitere Faktoren beachtet werden, die diese beeinflussen. Deshalb greifen wir in diesem Abschnitt auf multivariate Analysen zurück.

Im Zentrum des Interesses stehen Frauenerwerbsquote, Parlamentarierinnenquote und deren Zusammenspiel. Zunächst wurde der Zusammenhang dieser Faktoren mit den aktivierenden Sozialleistungen ermittelt. Die Ergebnisse der multivariaten Analysen sind in Tab. 2 dargestellt. ${ }^{12}$ Wie erwartet, wirken sich beide Variablen positiv auf die Höhe dieser Ausgaben aus (Modell 1). Im zweiten Modell wird zusätzlich die Interaktion zwischen diesen beiden Faktoren in das Modell aufgenommen. Aus dem positiven Koeffizienten geht hervor, dass sich die Frauenerwerbsquote stärker auf den Ausbau sozialinvestiver Leistungen auswirkt, wenn es viele weibliche Abgeordnete gibt. ${ }^{13}$ Diesen Zusammenhang verdeutlicht Abb. 3, in der die Effektstärke der Erwerbsbeteiligung von Frauen in Abhängigkeit von der Parlamentarierinnenquote dargestellt ist. Sobald etwa 10 Prozent der Abgeordneten Frauen sind, wirkt sich die Erwerbsquote positiv auf aktivierende Leistungen aus, und mit jeder weiteren Zunahme von Frauen in den Parlamenten wird dieser Effekt stärker. Dieses Ergebnis steht im Einklang mit der Annahme, dass die Forderungen nach Maßnahmen, die eine gleichberechtigte Teilnahme am Arbeitsmarkt ermöglichen, von weiblichen Abgeordneten stärker aufgegriffen und umgesetzt werden und bestätigt Theorien der „Gruppenrepräsentation“, die davon ausgehen, dass sich politische Entscheidungen dadurch verändern, wer im Parlament vertreten ist (siehe Phillips 1995).

Um auszuschließen, dass der Zusammenhang allein durch die nordischen Länder geprägt wird, fügt Modell 3 eine Dummy-Variable für die vier skandinavischen Länder hinzu. Zwar zeigt sich ein starker Effekt, ohne aber das Muster der anderen

\footnotetext{
11 Damit widersprechen unsere Ergebnisse Kuitto (2016), die jedoch nur europäische Ländern in den 2000er-Jahren betrachtet. Sie finden keinen Zielkonflikt zwischen aktivierenden und kompensatorischen Ausgaben.

12 Aus Platzgründen verzichten wir auf eine Diskussion der anderen Kontrollvariablen, die größtenteils die erwarteten Effekte aufweisen.

13 Die nun negativen Koeffizienten für Frauenerwerbsquote und Parlamentarierinnenquote sagen lediglich, dass eine der beiden Variablen sich negativ auswirkt, falls die andere den Wert „Null“ annimmt. Wenn die Variablen stattdessen zentriert werden, bleiben beide Vorzeichen positiv, weil nun ausgedrückt wird, wie der Effekt einer Variable ist, wenn die andere den mittleren Wert annimmt.
} 


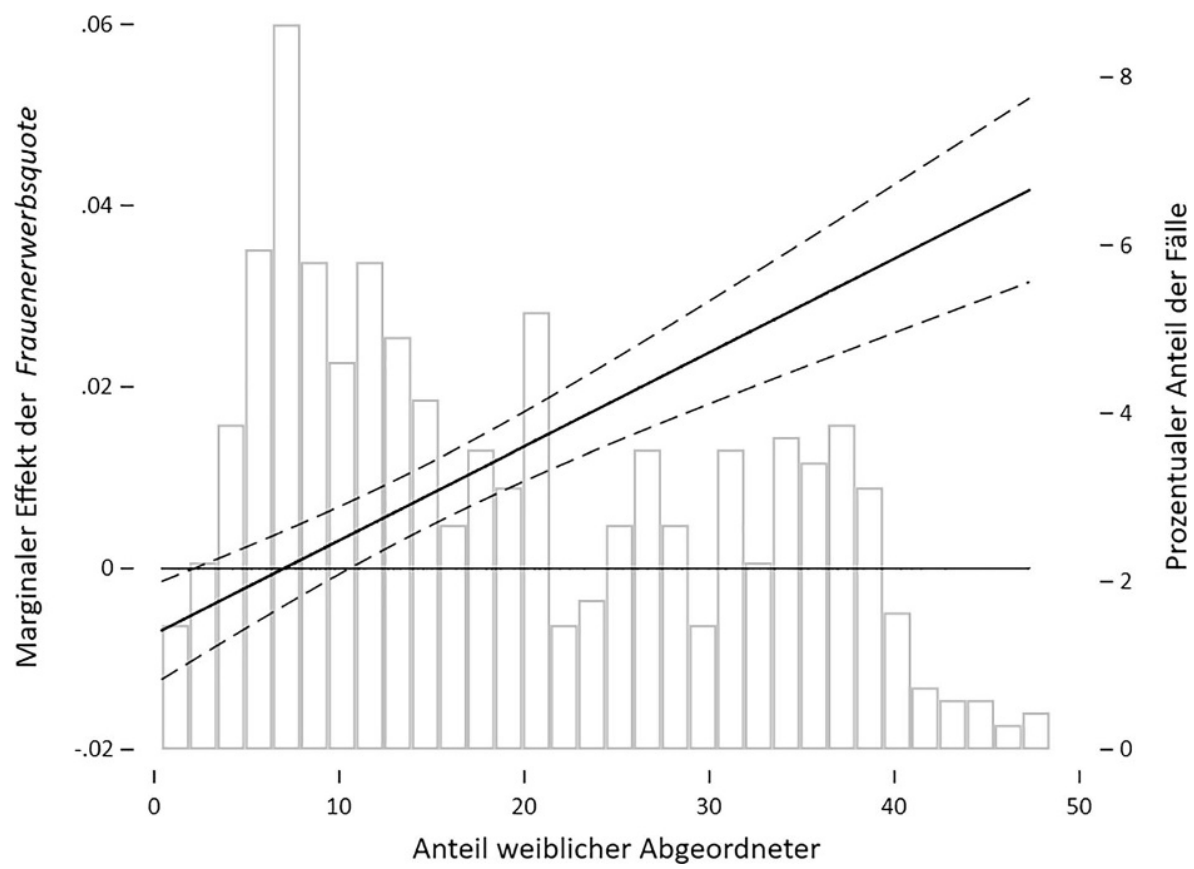

Abb. 3 Der Effekt der Frauenerwerbsquote auf aktivierende Sozialleistungen in Abhängigkeit von der Anzahl weiblicher Abgeordneter. Anmerkungen: Die Abbildung beruht auf Modell 3 in Tab. 2. Dargestellt ist der durchschnittliche marginale Effekt der Frauenerwerbsquote in Abhängigkeit von der Anzahl weiblicher Parlamentarier. Die unausgefüllten Balken geben den Anteil der Fälle wieder

Variablen zu verändern. Dasselbe gilt für den zeitlichen Trend. Zwar fand in den 1990er-Jahren ein stärkerer Ausbau als in den 1980er-Jahren statt, aber die im Mittelpunkt dieses Beitrags stehenden Variablen werden durch die Berücksichtigung dieses Faktors nicht beeinflusst. Die Robustheit der Ergebnisse bestätigen auch zwei weitere Tests, in denen ein Random Effects- und ein Fixed Effects-Modell geschätzt und mit Modell 3 aus der vorherigen Analyse verglichen werden (siehe Tabelle A-2 im Online-Anhang).

Ein im Vergleich zu den aktivierenden Sozialausgaben anderes Bild ergibt sich für die passiven Sozialleistungen. Zwar beeinflussen sich auch hier Frauenerwerbsquote und Frauenrepräsentation gegenseitig, aber je höher beide sind, desto niedriger fallen die Ausgaben aus. Im ersten Modell in Tab. 3 werden erneut die beiden zentralen erklärenden Variablen unabhängig voneinander eingeschlossen. Die Frauenerwerbsquote übt einen schwach negativen, aber nicht signifikanten Effekt auf die Höhe dieser Leistungen aus, während die Anzahl der Parlamentarierinnen einen signifikant positiven Effekt ausübt. Interessant ist erneut das zweite Modell, in dem ein Interaktionseffekt der beiden Variablen zusätzlich eingeführt wird. Wie sich Abb. 4 entnehmen lässt, wirkt sich eine hohe Frauenerwerbsquote negativ auf passive Leistungen aus, wenn gleichzeitig viele Parlamentarierinnen im Parlament vertreten sind. Auch dieses Ergebnis bleibt unter dem Einschluss weiterer Variablen (Modell 3) und bei anderen Schätzverfahren bestehen (siehe Tabelle A-3 im Online-Anhang). 
Tab. 3 Erklärung des Umfangs von passiven Sozialleistungen

\begin{tabular}{|c|c|c|c|}
\hline & Modell 1 & Modell 2 & Model 3 \\
\hline LDV & $\begin{array}{l}0,794 * * * \\
(0,02)\end{array}$ & $\begin{array}{l}0,773 * * * \\
(0,01)\end{array}$ & $\begin{array}{l}0,754 * * * \\
(0,02)\end{array}$ \\
\hline Frauenerwerbsquote & $\begin{array}{l}-0,006^{*} \\
(0,00)\end{array}$ & $\begin{array}{l}0,009 * \\
(0,00)\end{array}$ & $\begin{array}{l}0,013 * * \\
(0,00)\end{array}$ \\
\hline Parlamentarierinnen & $\begin{array}{l}0,012^{* * * *} \\
(0,00)\end{array}$ & $\begin{array}{l}0,092 * * * \\
(0,01)\end{array}$ & $\begin{array}{l}0,102 * * * \\
(0,02)\end{array}$ \\
\hline Erwerbsquote*Parlamentarierinnen & - & $\begin{array}{l}-0,001 \text { *** } \\
(0,00)\end{array}$ & $\begin{array}{l}-0,001 \text { *** } \\
(0,00)\end{array}$ \\
\hline Wahlbeteiligung & $\begin{array}{l}0,004 * \\
(0,00)\end{array}$ & $\begin{array}{l}0,002 \\
(0,00)\end{array}$ & $\begin{array}{l}0,006^{* * *} \\
(0,00)\end{array}$ \\
\hline Mitte-links-Regierungen & $\begin{array}{l}0,009 * * * \\
(0,00)\end{array}$ & $\begin{array}{l}0,015^{* * * *} \\
(0,00)\end{array}$ & $\begin{array}{l}0,012^{* * * *} \\
(0,00)\end{array}$ \\
\hline Gewerkschaftsdichte & $\begin{array}{l}0,001 \\
(0,00)\end{array}$ & $\begin{array}{l}0,004 * * * \\
(0,00)\end{array}$ & $\begin{array}{l}-0,003+ \\
(0,00)\end{array}$ \\
\hline Arbeitslosenquote & $\begin{array}{l}0,022^{* * *} \\
(0,01)\end{array}$ & $\begin{array}{l}0,020 * \\
(0,01)\end{array}$ & $\begin{array}{l}0,021 * \\
(0,01)\end{array}$ \\
\hline Anteil Über 65-Jährige & $\begin{array}{l}-0,008 \\
(0,01)\end{array}$ & $\begin{array}{l}-0,016 \\
(0,01)\end{array}$ & $\begin{array}{l}-0,016 \\
(0,01)\end{array}$ \\
\hline Anteil Unter-5-Jährige & $\begin{array}{l}0,098 * * * \\
(0,03)\end{array}$ & $\begin{array}{l}0,098 * * * \\
(0,03)\end{array}$ & $\begin{array}{l}0,097 * * * \\
(0,03)\end{array}$ \\
\hline Außenhandel (log.) & $\begin{array}{l}0,307 * * * \\
(0,04)\end{array}$ & $\begin{array}{l}0,283^{* * * *} \\
(0,05)\end{array}$ & $\begin{array}{l}0,429 * * * \\
(0,07)\end{array}$ \\
\hline BIP (log.) & $\begin{array}{l}-0,237 * * \\
(0,09)\end{array}$ & $\begin{array}{l}-0,305^{* * *} \\
(0,08)\end{array}$ & $\begin{array}{l}-0,268 * \\
(0,11)\end{array}$ \\
\hline Vetopunkte & $\begin{array}{l}0,024 * * \\
(0,01)\end{array}$ & $\begin{array}{l}0,023 * \\
(0,01)\end{array}$ & $\begin{array}{l}0,028 * \\
(0,01)\end{array}$ \\
\hline Skandinavien & - & - & $\begin{array}{l}0,558 * * * \\
(0,13)\end{array}$ \\
\hline \multicolumn{4}{|l|}{ Jahrzehnt (RK: 1980er-Jahre) } \\
\hline $1990 \mathrm{er}$ & - & - & $\begin{array}{l}0,088 \\
(0,10)\end{array}$ \\
\hline 2000er & - & - & $\begin{array}{l}-0,029 \\
(0,14)\end{array}$ \\
\hline Konstante & $\begin{array}{l}1,040 \\
(0,85)\end{array}$ & $\begin{array}{l}1,076 \\
(0,85)\end{array}$ & $\begin{array}{l}-0,048 \\
(1,19)\end{array}$ \\
\hline $\mathrm{R}^{2}$ & 0,88 & 0,88 & 0,88 \\
\hline$N$ & 591 & 591 & 591 \\
\hline
\end{tabular}

Standardfehler in Klammern. $+p<0,1, * p<0,05, * * p<0,01, * * * p<0,001$

Anmerkung: Dargestellt sind die Koeffizienten einer OLS-Regression mit Panel Corrected Standard Errors mit einer Prais-Winsten-Transformation (AR1), um für Autokorrelation in den Störtermen zu korrigieren. Alle Modelle schließen die Lagged Dependent Variable $(L D V)$ als unabhängige Variable ein.

Unsere Analysen führen somit zu einer Differenzierung bestehender Ergebnisse. Bei Bolzendahl und Brooks (2007) wurde ein positiver Effekt unserer beiden Hauptvariablen für die Höhe der gesamten Sozialausgaben ermittelt. Doch der Blick auf diese hochaggregierten Zahlen verdeckt, dass der Übergang zum Sozialinvestitionsstaat mit Kürzungen in einigen und Ausgabenzuwächsen in anderen Bereichen einhergeht. Die Analysen des vorangegangenen Abschnitts haben gezeigt, dass das 


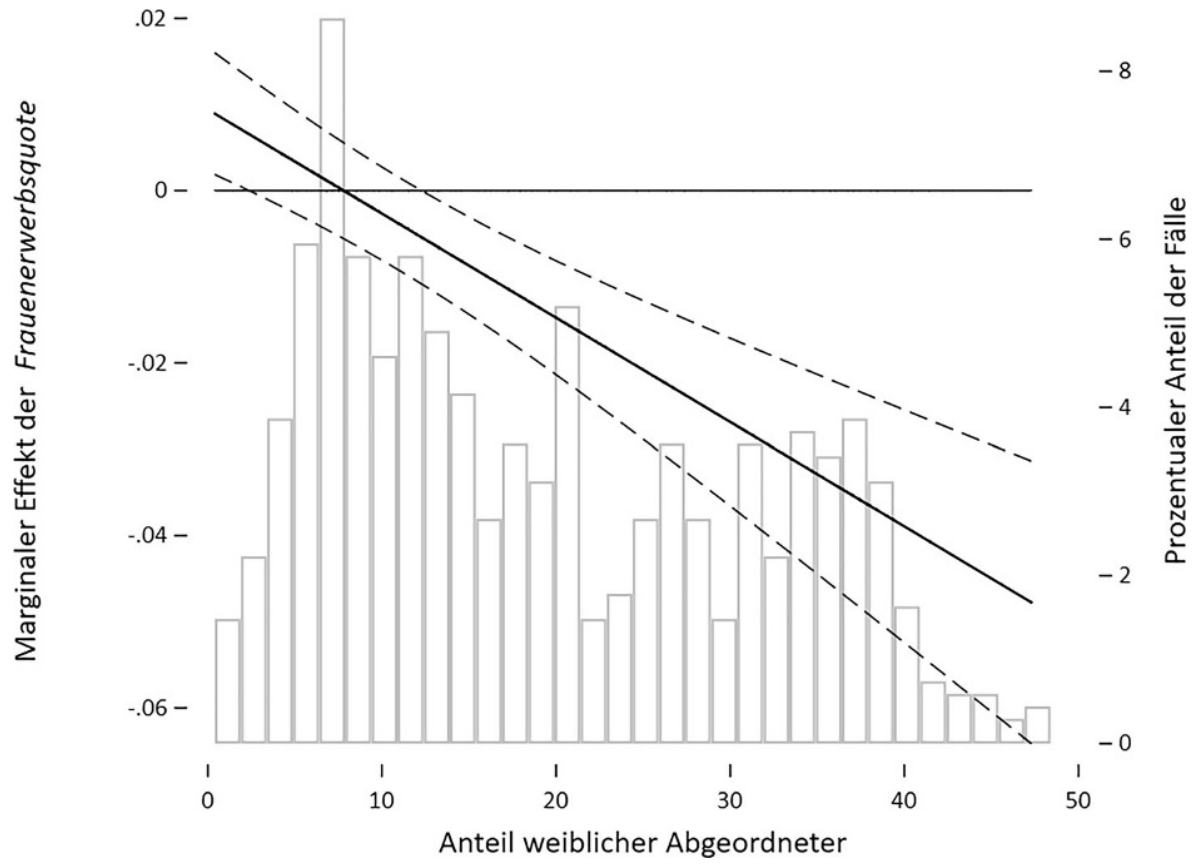

Abb. 4 Der Effekt der Frauenerwerbsquote auf passive Sozialleistungen in Abhängigkeit von der Anzahl weiblicher Abgeordneter. Anmerkungen: Die Abbildung beruht auf Modell 3 in Tab. 3. Dargestellt ist der durchschnittliche marginale Effekt der Frauenerwerbsquote in Abhängigkeit von der Anzahl weiblicher Parlamentarierinnen. Die unausgefüllten Balken geben den Anteil der Fälle wieder

Zusammenspiel einer höheren Erwerbsbeteiligung von Frauen mit einer besseren Repräsentation von (höhergebildeten) Frauen in den Parlamenten die Verlagerung von passiven zu aktivierenden Leistungen prägt. Während soziale Dienstleistungen ausgebaut werden, die die Vereinbarkeit von Familie und Beruf verbessern sollen, werden passive Leistungen gekürzt, die einerseits Arbeitslosen zugutekommen und andererseits klassische Familienformen stützen. Der Umbau des Sozialstaats weist damit einen Mittelschichtsbias auf, da die schwächsten Gruppen am wenigsten von den aktivierenden Sozialausgaben profitieren und gleichzeitig am stärksten von den Kürzungen bei den passiven Sozialausgaben betroffen sind. Gleichzeitig eröffnen sozialinvestive Maßnahmen dort, wo sie gut ausgebaut und ausgestaltet sind, Frauen die Möglichkeit, sich nicht zwischen Kindern und Lohnarbeit entscheiden zu müssen und fördern dadurch unter anderem die finanzielle Unabhängigkeit von einem Partner und/oder dem Staat. Inwiefern aktive Sozialpolitik Geschlechtergerechtigkeit (horizontale Umverteilung) über alle Schichten hinweg fördert oder hauptsächlich dazu beiträgt, Frauen in schlecht bezahlte Jobs zu zwingen, hängt also von der 
Ausgestaltung aktiver Sozialpolitik ab (Orloff 2006, S. 232-233). ${ }^{14}$ Allerdings ist in vielen Ländern zu beobachten, dass es vor allem Frauen aus mittleren und oberen Schichten sind, denen sich durch diese Maßnahmen Möglichkeiten eröffnen (Pavolini und Van Lancker 2018; Van Lancker 2013).

\section{Diskussion der Ergebnisse}

Für keinen Politikbereich geben entwickelte Demokratien mehr aus als für die Sozialpolitik. Denn die Bürgerinnen und Bürger sehen den Staat in der Pflicht, sie vor den Folgen sozialer Risiken wie Arbeitslosigkeit, Krankheit oder Pflegebedürftigkeit im Alter zu schützen. Da durch den Übergang zur Dienstleistungsgesellschaft, durch den Wandel der Geschlechterrollen und die Globalisierung die Ansprüche an den Sozialstaat weiter gestiegen sind, während die Haushaltslage in vielen Ländern angespannt ist, geraten Regierungen unter Handlungsdruck. Sie reagieren darauf, so haben wir in diesem Aufsatz gezeigt, durch die Kombination von sozialen Einschnitten und Ausgabensteigerungen. Wo mehr ausgeben und wo gekürzt wird, folgt dabei keinem zufälligen Muster, sondern hängt auch mit deskriptiver politischer Repräsentation zusammen.

Wie wir in diesem Beitrag gezeigt haben, lässt sich zumindest auf aggregierter Ebene ein Zusammenhang zwischen der Repräsentation von Frauen in Parlamenten und dem Umbau des Sozialstaats nachweisen. Dort, wo der Anteil weiblicher Abgeordneter hoch ist und gleichzeitig viele Frauen am Arbeitsmarkt partizipieren, geben Staaten mehr für Maßnahmen zur Vereinbarkeit von Familie und Beruf und anderen aktivierende Dienstleistungen aus. Die hier zugrunde gelegte Annahme ist, dass eine erhöhte Frauenerwerbstätigkeit den Bedarf an sozialen Dienstleistungen erhöht und insbesondere Frauen diese politisch nachfragen. Gleichzeitig werden in eben diesen Staaten passive Sozialleistungen, die vor traditionellen Risiken schützen, stärker zurückgebaut. Daraus lässt sich jedoch nicht ableiten, dass es Parlamentarierinnen waren, die sich besonders energisch für die Kürzung von kompensatorischen Leistungen eingesetzt haben. Vielmehr könnte die stärkere deskriptive politische Repräsentation von (Mittelschichts-)Frauen dazu geführt haben, dass trotz Einsparungsdruck Teile ihrer sozialpolitischen Anliegen umgesetzt wurden, wohingegen für andere Anliegen, wie dem Schutz vor traditionellen Risiken wie Arbeitslosigkeit, keine Mehrheiten zu finden waren. Denn die veränderte Schwerpunktsetzung hin zum Sozialinvestitionsstaat fand zu einem Zeitpunkt statt, zu dem die Expansionsphase des Sozialstaats bereits beendet war und der Druck stieg, Einsparungen vorzunehmen. Der Ausbau einer Infrastruktur, die die bessere Vereinbarkeit von Familie und Beruf ermöglicht, versprach, sich zumindest in Teilen durch höhere Erwerbsquoten zu refinanzieren, wohingegen dieser Effekt bei passiven Leistungen

\footnotetext{
$14 \mathrm{Ob}$ Geschlechtergerechtigkeit verwirklicht werden kann, hängt natürlich nicht nur von staatlichen Maßnahmen, sondern auch von Rollenveränderungen und dem Verhalten der Partner ab. Allerdings ist auch hier zu beobachten, dass Männer aus höheren Bildungsschichten und in Partnerschaften, in denen Frauen gut bezahlte Jobs haben, tendenziell einen größeren Beitrag zur Haus- und Sorgearbeit leisten (EspingAndersen 2009, S. 99-104), was die beschriebenen Muster verstärkt.
} 
nicht zu erwarten war. Darüber hinaus ließ sich nicht immer vorhersehen, wer von neuen Leistungen profitieren würde.

Unsere Analysen bestätigten, dass die Zusammensetzung der Parlamente für die sozialpolitischen Ausgabenprofile der untersuchten Länder relevant ist. Doch während die Legislativen mit Blick auf das Geschlecht heterogener als früher sind, sind sie in sozialer Hinsicht homogener geworden. Während Arbeiterinnen und Arbeiter kaum noch in den Parlamenten vertreten sind, geht die Unterrepräsentation von Frauen - aus der Mittelschicht - zurück (Best 2007; Carnes 2012; Wiesendahl 2017). Bovens und Wille (2017) sprechen von „Diploma Democracy“, um die Dominanz von Akademikerinnen und Akademikern in den Parlamenten zu verdeutlichen. Im Zuge dieses Repräsentationswandelns beobachten wir, dass der Sozialstaat stärker an horizontaler Gerechtigkeit ausgerichtet wird, wohingegen die Leistungen für sozial benachteiligte Frauen und Männer eher gekürzt werden. Denn untere soziale Schichten profitieren weit weniger von den neuen familienpolitischen Maßnahmen (Abrassart und Bonoli 2015; OECD 2011; Pavolini und Van Lancker 2018; Van Lancker 2013). Vor allem beim Zugang zu und der Inanspruchnahme von Kinderbetreuung spielt die soziale Schicht eine entscheidende Rolle. In allen EU-Staaten werden Kinderbetreuungseinrichtungen weitaus häufiger von Eltern aus hohen Einkommensschichten als von Eltern mit niedrigem Einkommen genutzt - nur in Dänemark und Schweden ist die Inanspruchnahme ähnlich. In den osteuropäischen EU-Staaten und Frankreich dagegen ist die Ungleichheit in der Betreuung besonders hoch (Van Lancker 2013, S. 14). Auch von anderen familienpolitischen Maßnahmen zur Vereinbarkeit von Familie und Beruf, wie etwa bezahlte Elternzeit, profitieren höhere Schichten stärker, wie Ghysels und van Lancker (2011) in ihrer Untersuchung verschiedener familienpolitischer Maßnahmen am Beispiel Belgiens zeigen. ${ }^{15}$ Einer der Gründe für diese Befunde ist, dass Frauen aus unteren Bildungs- und Einkommensschichten weiterhin weniger am Arbeitsmarkt partizipieren als Frauen aus höheren Schichten (Evertsson et al. 2009) und allein deshalb weniger Gebrauch dieser Programme machen. Insgesamt gehören Doppelverdienerhaushalte weitaus häufiger zur Mittel- oder Oberschicht, weshalb diese tendenziell stärker von Maßnahmen zur Vereinbarkeit von Familie und Beruf profitieren (Cantillon 2011, S. 441-442; Esping-Andersen 2009, S. 66-70). Da Paare heute zudem deutlich häufiger aus ähnlichen Schichten kommen, verstärken diese Entwicklungen den Trend steigender Einkommensungleichheit (Esping-Andersen 2009, Kap. 2). Cantillon (2011) zeigt zudem, dass sozialinvestive Maßnahmen bisher nicht zur Inklusion und Aktivierung der ärmsten Haushalte geführt haben: Die Gruppe der Haushalte, in denen kein Mitglied arbeitet, bleibt konstant. Gleichzeitig hat das Armutsrisiko von Haushalten, die wenig oder gar nicht am Arbeitsmarkt partizipieren, zugenommen.

\footnotetext{
15 In ihrer intersektionalen Analyse familienpolitischer Reformen in Deutschland argumentieren Menke und Klammer (2017) in Bezug auf die Einführung des Elterngeldes ähnlich. So begünstigt die Kopplung des Elterngeldes an die Erwerbseinkommen besonders stark diejenigen Eltern, die sich erfolgreich am Arbeitsmarkt positioniert haben. Dagegen wird Arbeitslosengeld-II-Empfängerinnen und -Empfängern das Elterngeld auf die Sozialleistungen angerechnet, was beim vorher existierenden Erziehungsgeld nicht der Fall war. Somit kam die Einführung des Elterngeldes für Mütter und Väter im Arbeitslosengeld II-Bezug einer Leistungskürzung von passiven Transfers gleich.
} 
Die „Politik der Anwesenheit“, so legen unser Ergebnisse nahe, ist nicht nur für Frauen, sondern auch für soziale Klassen relevant (siehe die Diskussion bei Mansbridge 2015). Um die kausalen Mechanismen hinter den aufgezeigten Mustern näher zu erforschen, wären jedoch ergänzende, auf der Mikroebene angesiedelte Forschungsarbeiten notwendig, die das Abstimmungsverhalten bzw. das AgendaSetting verschiedener sozialer Gruppen in Parlamenten untersuchen, wodurch und von wem sozialpolitische Prioritätensetzung vorgenommen wird (siehe bspw. Carnes 2012). Eine detaillierte Analyse einzelner Reformen würde es ermöglichen, die Frage zu beantworten, ob die Ausweitung sozialinvestiver Maßnahmen in politischen Verhandlungen explizit gegen Kürzungen in anderen Bereichen abgewogen wurde und ob die soziale Zusammensetzung des Parlaments zu einer stärkeren Mittelschichtsorientierung führt.

Danksagung Für hilfreiche Kommentare danken wir Sebastian Huhnholz, Daniel Mertens, Thomas Rixen, Laura Seelkopf sowie drei anonymen Gutachterinnen und Gutachtern der PVS.

\section{Literatur}

Abrassart, Aurelien, und Giuliano Bonoli. 2015. Availability, cost or culture? Obstacles to childcare services for low-income families. Journal of Social Policy 44(04):787-806.

Achen, Christopher. 2000. Why lagged dependent variables can suppress the explanatory power of other independent variables. Los Angeles. Paper prepared for delivery at the Political Methodology Summer Meeting, 20-22.

Armingeon, Klaus, Christian Isler, Laura Knöpfel, David Weisstanner, und Sarah Engler. 2016. Comparative Political Data Set 1960-2014. Bern: Institute of Political Science, University of Bern.

Beck, Nathaniel. 2001. Time-series cross-section data: what have we learned in the past few years? Annual Review of Political Science 4(1):271-293. https://doi.org/10.1146/annurev.polisci.4.1.271.

Beck, Nathaniel, und Jonathan N. Katz. 1995. What to Do (and Not to Do) with Time-Series Cross-Section Data. American Political Science Review 89(3):634-647.

Best, Heinrich. 2007. New challenges, new elites? Changes in the recruitment and career patterns of European representative elites. Comparative Sociology 6:85-113.

Bolzendahl, Catherine. 2011. Beyond the big picture: gender influences on disaggregated and domainspecific measures of social spending, 1980-1999. Politics \& Gender 7(1):35-70.

Bolzendahl, Catherine, und Clem Brooks. 2007. Women's political representation and welfare state spending in 12 capitalist democracies. Social Forces 85(4):1509-1534.

Bonoli, Giuliano. 2007. Time matters: postindustrialization, new social risks, and welfare state adaptation in advanced industrial democracies. Comparative Political Studies 40(5):495-520.

Bonoli, Giuliano. 2013. The origins of active social policy: labour market and Childcare policies in a comparative perspective. Oxford: Oxford University Press.

Bovens, Mark A. P., und Anchrit Wille. 2017. Diploma democracy: the rise of political meritocracy. Oxford: Oxford University Press.

Bratton, Kathleen A., und Leonard P. Ray. 2002. Descriptive representation, policy outcomes, and municipal day-care coverage in Norway. American Journal of Political Science 46(2):428-437.

Brunsbach, Sandra. 2011. Machen Frauen den Unterschied? Parlamentarierinnen als Repräsentatinnen frauenspezifischer Interessen im Deutschen Bundestag. Zeitschrift für Parlamentsfragen 42:3-24.

Busemeyer, Marius R., Caroline de la Porte, Julian L. Garritzmann, und Emmanuele Pavolini. 2018. The future of the social investment state: politics, policies, and outcomes. Journal of European Public Policy 25(6):801-809. https://doi.org/10.1080/13501763.2017.1402944.

Cantillon, Bea. 2011. The paradox of the social investment state: growth, employment and poverty in the Lisbon Era. Journal of European Social Policy 21(5):432-449.

Carnes, Nicholas. 2012. Does the numerical underrepresentation of the working class in congress matter? Legislative Studies Quarterly XXXVII 1/2012:5-34. https://doi.org/10.1111/j.1939-9162.2011.00033. $\mathrm{X}$. 
Castles, Francis G. 2009. What welfare states do: a disaggregated expenditure approach. Journal of Social Policy 38(01):45-62.

Elsässer, Lea, Inga Rademacher, und Armin Schäfer. 2015. Cracks in the foundations. Retrenchment in advanced welfare states. economic sociology 16:4-16.

Esping-Andersen, Gøsta. 1990. The three worlds of welfare capitalism. Cambridge: Polity Press.

Esping-Andersen, Gøsta. 1999. New social risks in old welfare states. In Social foundations of Postindustrial societies, Hrsg. Gosta Esping-Andersen, 145-169. Oxford: Oxford University Press.

Esping-Andersen, Gøsta. 2002. Why we need a new welfare state. Oxford: Oxford University Press.

Esping-Andersen, Gøsta. 2009. The incomplete revolution. Adapting the welfare state to women's new roles. London: Polity Press.

Evertsson, Marie, Paula England, Irma Mooi-Reci, Joan Hermsen, Jeanne De Bruijn, und David Cotter. 2009. Is gender inequality greater at lower or higher educational levels? Common patterns in the Netherlands, Sweden, and the United States. Social Politics: International Studies in Gender, State \& Society 16(2):210-241.

Ferragina, Emanuele, und Martin Seeleib-Kaiser. 2015. Determinants of a silent (R)evolution: understanding the expansion of family policy in rich OECD countries. Social Politics: International Studies in Gender, State \& Society 22(1):1-37.

Finseraas, Henning, Niklas Jakobsson, und Andreas Kotsadam. 2012. The gender gap in political preferences: an empirical test of a political economy explanation. Social Politics 19(2):219-242.

Ghysels, Joris, und Wim Van Lancker. 2011. The unequal benefits of activation: an analysis of the social distribution of family policy among families with young children. Journal of European Social Policy 21(5):472-485.

Giger, Nathalie. 2009. Towards a modern gender gap in Europe? A comparative analysis of voting behavior in 12 countries. The Social Science Journal 46:474-492.

Gingrich, Jane, und Ben W. Ansell. 2015. The dynamics of social investment: human capital, activation, and care. In The politics of advanced capitalism, Hrsg. Pablo Beramendi, Silja Häusermann, Herbert Kitschelt, und Hanspeter Kriesi, 282-304. Cambridge: Cambridge University Press.

Grey, Sandra. 2006. Numbers and beyond: the relevance of critical mass in gender research. Politics \& Gender 2(4):492-502.

Huber, Evelyne, und John D. Stephens. 2000. Partisan governance, women's employment, and the social democratic service state. American Sociological Review 65(3):323-342.

Huber, Evelyne, und John D. Stephens. 2014. Income inequality and redistribution in post-industrial democracies: demographic, economic and political determinants. Socio-Economic Review 12(2):245-267.

Inglehart, Ronald, und Pippa Norris. 2003. Rising tide: gender equality and cultural change around the world. Cambridge: Cambridge University Press.

Iversen, Torben, und Frances Rosenbluth. 2006. The political economy of gender: explaining cross-national variation in the gender division of labor and the gender voting gap. American Journal of Political Science 50(1):1-19.

Jenson, Jane. 2015. The fading goal of gender equality: three policy directions that underpin the resilience of gendered socio-economic inequalities. Social Politics: International Studies in Gender, State \& Society 22(4):539-560.

Kittel, Bernhard. 1999. Sense and sensitivity in pooled analysis of political data. European Journal of Political Research 35:225-253.

Kittilson, Miki C. 2008. Representing women: the adoption of family leave in comparative perspective. The Journal of Politics 70(2):323-334.

Kuitto, Kati. 2016. From social security to social investment? Compensating and social investment welfare policies in a life-course perspective. Journal of European Social Policy 26(5):442-459.

Van Lancker, Wim. 2013. Putting the child-centered investment strategy to the test: evidence for the EU27. European Journal of Social Security 15:4-27.

Mansbridge, Jane. 2015. Should workers represent workers? Swiss Political Science Review 21(2):261-270.

Menke, Katrin, und Ute Klammer. 2017. Mehr Geschlechtergerechtigkeit - weniger soziale Gerechtigkeit? Familienpolitische Reformprozesse in Deutschland aus intersektionaler Perspektive. Sozialer Fortschritt 66:213-228.

Meyer, Birgit. 2003. Much Ado about nothing? Political representation policies and the influence of women parliamentarians in Germany. Review of Policy Research 20(3):401-422.

Morgan, Kimberly J. 2013. Path shifting of the welfare state: electoral competition and the expansion of work-family policies in Western Europe. World Politics 65(1):73-115. 
Nikolai, Rita. 2012. Towards social investment? Patterns of public policy in the OECD world. In Towards a social investment welfare state? Ideas, policies and challenges, Hrsg. Nathalie Morel, Bruno Palier, und Joakim Palme, 91-116. Bristol: The Policy Press.

OECD. 2011. Doing better for families. Paris: OECD Publishing.

OECD. 2016. Social expenditure: aggregated data. OECD.Stat, https://stats.oecd.org/Index.aspx? DataSetCode=SOCX_AGG.

Orloff, Ann Shola. 2006. From maternalism to 'employment for all. In The state after statism - new state activities in the age of liberalization, Hrsg. Jonah D. Levy, 230-268. Cambridge: Harvard University Press.

Palier, Bruno. 2006. The re-orientation of Europe social policies towards social investment. International Journal of Politics, Culture and Society 1:105-116.

Pavolini, Emmanuele, und Wim Van Lancker. 2018. The Matthew effect in childcare use: a matter of policies or preferences? Journal of European Public Policy 25(6):878-893. https://doi.org/10.1080/ 13501763.2017.1401108.

Phillips, Anne. 1995. The politics of presence. The political representation of gender, ethnicity, and race. Oxford: Oxford University Press.

Pierson, Paul. 2001. Coping with permanent austerity. Welfare state restructuring in affluent democracies. In The new politics of the welfare state, Hrsg. Paul Pierson, 410-456. Oxford: Oxford University Press.

Pintelon, Olivier, Bea Cantillon, Karel Van den Bosch, und Christopher T. Whelan. 2013. The social stratification of social risks: the relevance of class for social investment strategies. Journal of European Social Policy 23(1):52-67.

Plümper, Thomas, Vera E. Troeger, und Philip Manow. 2005. Panel data analysis in comparative politics: linking method to theory. European Journal of Political Research 44:327-354.

Taylor-Gooby, Peter. 2004. New risks and social change. In New risks, new welfare. The transformation of the European welfare state, Hrsg. Peter Taylor-Gooby, 1-28. Oxford: Oxford University Press.

Tepe, Markus, und Pieter Vanhuysse. 2010. Elderly bias, new social risks and social spending: change and timing in eight programmes across four worlds of welfare, 1980-2003. Journal of European Social Policy 20(3):217-234.

Van Kersbergen, Kees, und Anton Hemerijck. 2012. Two decades of change in Europe: the emergence of the social investment state. Journal of Social Policy 41(03):475-492.

Wängnerud, Lena. 2000. Testing the politics of presence: women's representation in the Swedish riksdag. Scandinavian Political Studies 23(1):67-91.

Wängnerud, Lena. 2009. Women in parliaments: descriptive and substantive representation. Annual Review of Political Science 12(1):51-69. https://doi.org/10.1146/annurev.polisci.11.053106.123839.

Wiesendahl, Elmar. 2017. Parteien in gehobener Gesellschaft oder die halbierte Demokratie. In Parteien und soziale Ungleichheit, 413-439. Wiesbaden: Springer VS.

Wilson, Sven E., und Daniel M. Butler. 2017. A lot more to do: the sensitivity of time-series cross-section analyses to simple alternative specifications. Political Analysis 15(2):101-123. 